\title{
Infinite groups with many permutable subgroups
}

\section{A. Ballester-Bolinches, L. A. Kurdachenko, J. Otal and T. Pedraza}

\begin{abstract}
A subgroup $H$ of a group $G$ is said to be permutable in $G$, if $H K=K H$ for every subgroup $K$ of $G$. A result due to Stonehewer asserts that every permutable subgroup is ascendant although the converse is false. In this paper we study some infinite groups whose ascendant subgroups are permutable ( $A P$-groups). We show that the structure of radical hyperfinite $A P$-groups behave as that of finite soluble groups in which the relation to be a permutable subgroup is transitive ( $P T$-groups).
\end{abstract}

\section{Introduction}

A subgroup $H$ of a group $G$ is said to be permutable in $G$ (or quasi-normal in $G$ ), if $H K=K H$ for every subgroup $K$ of $G$. This concept arises as a generalization of that of normal subgroup. The study of the properties of the permutable subgroups started a rather long time ago (see, for example [17]). In particular, the groups (finite and infinite) in which every subgroup is permutable have been described (see $[17,2.4]$ ). Like normality, the relation to be a permutable subgroup is not transitive.

A subgroup $H$ of a group $G$ is said to be an ascendant subgroup of $G$ if there exists an ascending series from $H$ to $G$, that is, a chain of subgroups well-ordered by inclusion and indexed by the corresponding ordinal numbers

$$
H=H_{0} \triangleleft H_{1} \triangleleft \cdots \triangleleft H_{\alpha} \triangleleft H_{\alpha+1} \triangleleft \cdots \triangleleft H_{\gamma}=G
$$

with the additional stipulation that for each limit ordinal $\lambda$ the subgroup $H_{\lambda}$ is the union of all subgroups $H_{\beta}, \beta<\lambda$. This is an evident generalization of the concept of subnormal subgroup.

2000 Mathematics Subject Classification: 20F99.

Keywords: Radical groups, hyper- $\mathfrak{X}$-groups, $A P$-groups, $P T$-groups. 
According to a well-known theorem by Stonehewer ([18, Theorem A]), permutable subgroups are always ascendant. Therefore it is natural to consider the opposite situation, that is the groups whose ascendant subgroups are permutable. A group $G$ is said to be an $A P$-group if every ascendant subgroup of $G$ is permutable in $G$. These groups are very close to the groups in which the relation to be a permutable subgroup is transitive (studied in [3] for a class of periodic radical groups). A group $G$ is said to be a $P T$-group if permutability is a transitive relation in $G$, that is, if $K$ is a permutable subgroup of $H$ and $H$ is a permutable subgroup of $G$, then $K$ is a permutable subgroup of $G$. We note that, if $G$ is an $A P$-group, then clearly $G$ is a $P T$-group, because the relation to be an ascendant subgroup is transitive. If $G$ is a finite group, then it is known that $G$ is a $P T$-group if and only if every subnormal subgroup is permutable. The study of finite $P T$-groups began with a paper of G. Zacher [19]. Zacher determined the structure of finite soluble $P T$-groups in a similar way to Gaschütz's [7] characterization of finite soluble groups in which normality is a transitive relation (i.e. finite soluble T-groups).

Theorem (Zacher) Let $G$ be a finite soluble group. Then $G$ is a PT-group (and hence an AP-group) if and only if it has an abelian normal Hall subgroup $L$ of odd order such that $G / L$ is a nilpotent modular group and every subgroup of $L$ is normal in $G$.

As a consequence of this theorem, the class of finite soluble $P T$-groups (and therefore the class of finite soluble $A P$-groups) is subgroup-closed. There are many papers, in which several properties of finite soluble $P T$ groups have been considered (see, for example, [1, 4, 2]).

In [15, Lemma 4], it is claimed that in an arbitrary PT-group every ascendant subgroup is permutable. However this result is not true. An specific counterexample is the following. Let $G=A \rtimes\langle b\rangle$ the semidirect product of a Prüfer 2-group $A=\left\langle a_{n} \mid a_{1}^{2}=1, a_{n+1}^{2}=a_{n}, n \in \mathbb{N}\right\rangle$ by a cyclic group $\langle b\rangle$ of order 2 that acts on $A$ by $a^{b}=a^{-1}$ for each $a \in A$. If $x=a b$, for some $a \in A$, then $x^{2}=a b a b=a a^{-1}=1$. Let $H$ be a proper subgroup of $G$. If $H$ lies in $A$, then $H$ is $G$-invariant. Otherwise, $H$ is finite and $H=K\langle x\rangle$, where $K$ is a proper subgroup of $A, x \notin A$, and hence $|x|=2$. Every subgroup of $A$ is cyclic, so $K=\langle c\rangle$, where $c \in A$. Suppose that $|c|=2^{m}$, for some $m \in \mathbb{N}$. Then $|H|=2^{m+1}$. Since $A$ is divisible, we may choose an element $d \in A$ such that $d^{t}=c$, where $t=2^{5}$. Put $y=d x$. Clearly $y \notin A$, and thus $|y|=2$.

Suppose that $H\langle y\rangle=\langle y\rangle H$. Then $|H\langle y\rangle|=2^{m+1} \cdot 2=2^{m+2}$. On the other hand, $(d x) x \in\langle H, y\rangle$ and $(d x) x=d$, but $|d|=2^{m+5}$. This shows that $H$ can not be permutable in $G$. In other words, if $H$ is a permutable 
subgroup of $G$, then $H \leq A$, and in this case every permutable subgroup of $G$ is $G$-invariant. It follows that $G$ is a $P T$-group. However, the subgroup $\langle b\rangle$ is ascendant in $G$ but it is not permutable in $G$.

This example shows that for infinite groups the classes of $A P$-groups and $P T$-groups do not coincide. In this paper we start the study of infinite $A P$ groups. Obviously groups with no permutable subgroups are $A P$-groups, but it appears unreasonable to study properties of permutable subgroups in groups with no permutable subgroups. This shows that the effective study of $A P$-groups must be carried out under the imposition of some natural restrictions.

The natural framework for considering $A P$-groups are the classes of groups that have many ascendant subgroups. If $G$ is a nilpotent group, then every subgroup of $G$ is subnormal. Hence every subgroup of a nilpotent $A P$-group is permutable. A natural extension of the class of nilpotent groups is the class of locally nilpotent groups. One of the classes of locally nilpotent groups with many ascendant subgroups is the class of $N$-groups. We recall that a group $G$ is said to be an $N$-group, or a group with the normalizer condition, if every proper subgroup of $G$ is properly contained in its normalizer (see $[12, \S 63]$ ). It is worth mentioning that $G$ is a $N$-group if and only if every subgroup of $G$ is ascendant in $G([12, \S 63])$. A generalization of the class of $N$-groups is the class of Gruenberg groups. We recall that a group $G$ is said to be a Gruenberg group if every finitely generated subgroup of $G$ is ascendant in $G$. Every countable locally nilpotent group is a Gruenberg group, but there exists an uncountable locally nilpotent group which does not include ascendant finitely generated subgroups (see [10]). Furthermore, O. Yu. Schmidt [16] has constructed an example of a countable locally nilpotent $p$-group which is not an $N$-group. Being countable, this group is a Gruenberg group. This example shows that the classes of $N$-groups and Gruenberg groups are distinct. As we will see in section 2, every Sylow $p$-subgroup of a Gruenberg $A P$-group is nilpotent.

The following classes of generalized soluble groups spring from the above classes of locally nilpotent groups in same way the the class of finite soluble groups does from the class of finite nilpotent groups. Let $\mathfrak{X}$ be a class of groups. A group $G$ is said to be a hyper- $\mathfrak{X}$-group if there is an ordinal $\gamma$ such that $G$ has an ascending normal series of subgroups

$$
1=D_{0} \triangleleft D_{1} \triangleleft \cdots \triangleleft D_{\alpha} \triangleleft D_{\alpha+1} \triangleleft \cdots \triangleleft D_{\gamma}=G
$$

(where $D_{\lambda}=\bigcup_{\beta<\lambda} D_{\beta}$ if $\lambda$ is a limit ordinal) whose factors are $\mathfrak{X}$-groups.

In particular, if $\mathfrak{X}$ is the class of all locally nilpotent groups, then we obtain the class of radical groups (so called hyper-locally nilpotent-groups) which is a natural extension of the class of finite soluble groups. 
Our aim in this paper is to extend results about finite soluble $P T$-groups to some classes of infinite groups. We work within the class of hyperfinite groups. There we find some interesting subclasses as $A P$-hypernilpotent groups, $A P$-hyper-Gruenberg groups and $A P$-hyper- $N$-groups that are different extensions of the class of finite soluble $A P$-groups.

The layout of the paper is as follows. In section 2, some auxiliary results are proved. In section 3 , we give a full characterization of radical hyperfinite $A P$-groups in the following way.

Theorem A (Theorem 3.5). Let $G$ be a radical hyperfinite AP-group. Then the following assertions hold:

(i) $G$ is metabelian;

(ii) if $R$ is the locally nilpotent radical of $G$, then $R=L \times Z$, where $L$ is the locally nilpotent residual of $G$ and $Z$ is the upper hypercenter of $G$;

(iii) the Sylow $p$-subgroup of $L$ is the Sylow $p$-subgroup of $G$ for every prime $p$ occurring as a divisor of some element of $L$;

(iv) every subgroup of $L$ is $G$-invariant; and

(v) $G / L$ is hypercentral and every subgroup of $G / L$ is permutable.

Moreover, if the factor-group $G / L$ is countable, then $G$ splits over $L$. In particular, if $G$ is countable, then $G$ splits over $L$.

On the other hand, if $G$ is a periodic group having a normal abelian subgroup $L$ that satisfies the conditions (iii)-(v), then $G$ is an AP-group.

In particular, a radical hyperfinite group $G$ is an AP-group if and only if $G$ satisfies the conditions (iii)-(v) of Theorem $A$.

Theorem $\mathrm{A}$ has the virtue of showing that to be an AP-group is a subgroup-closed property in the universe of all radical hyperfinite groups, a fact that is not evident from the definition. Moreover it has another not less important virtue: it allows us to construct all periodic soluble $A P$-groups (see Section 5).

Corollary B (Corollary 3.6). Let $G$ be a radical hyperfinite AP-group. Then every subgroup of $G$ is a AP-group.

The above results confirm that the structure of (infinite) radical hyperfinite $A P$-groups is very similar to the structure of finite soluble $P T$-groups.

We finally analyze in Section 4 the structure of $A P$-groups in some classes of groups that are larger than the class of radical hyperfinite groups.

Theorem C (Theorem 4.4). Let $G$ be a periodic AP-group. If $G$ is a hyper-N-group, then $G$ is hyperfinite. 
Corollary D. Let $G$ be a periodic AP-group. If $G$ is a hyper-N-group, then $G$ is a hypercyclic metabelian AP-group.

Corollary E. Let $G$ be a periodic AP-group. If $G$ is a hyper-Gruenberg group, then $G$ is a hypercyclic metabelian $A P$-group. In particular, if $G$ is a countable radical group, then $G$ is a hypercyclic metabelian AP-group.

Corollary F. Let $G$ be a periodic AP-group. If $G$ is hyperabelian, then $G$ is a hypercyclic metabelian AP-group.

Corollary G. Let $G$ be a periodic AP-group. If $G$ is soluble, then $G$ is a hypercyclic metabelian AP-group.

Corollary H. Let $G$ be a periodic AP-group. If $G$ is residually soluble, then $G$ is a hypercyclic metabelian AP-group.

Our group-theoretical terminology is standard and can be found in [14] for example.

\section{Preliminary properties of $A P$-groups}

We start by establishing some useful facts. The first one is obvious.

Lemma 2.1 Let $G$ be an AP-group. Then $G / H$ is an AP-group for each normal subgroup $H$ of $G$.

Lemma 2.2 Let $G$ be an AP-group. Then every ascendant subgroup of $G$ is an $A P$-group. In particular, every subnormal subgroup of $G$ is an $A P-$ group.

Proof. Indeed, let $A$ be an ascendant subgroup of $G$. If $B$ is an ascendant subgroup of $A$, then $B$ is ascendant in $G$. Therefore $B$ is permutable in $G$ and hence in $A$.

Corollary 2.3 Let $G$ be an $A P$-group and let $A$ be an ascendant subgroup of $G$. Then $A / B$ is an $A P$-group for each subgroup $B$ which is normal in $A$.

Corollary 2.4 Let $G$ be an AP-group, $A$ an ascendant subgroup of $G$ and let $B$ a normal subgroup of $A$. If $A / B$ is a $N$-group, then every subgroup of $A / B$ is permutable.

As usual, if $G$ is a group, we denote by $\Pi(G)$ the set of all primes occurring as divisors of the order of some periodic element of $G$.

Corollary 2.5 Let $G$ be a periodic AP-group. If $G$ is a $N$-group, then $G$ can be expressed as a direct product

$$
G=D r_{p \in \Pi(G)} G_{p}
$$

where $G_{p}$ is the Sylow p-subgroup of $G$ and the following conditions holds: 
(1) if $p \neq 2$, then either $G_{p}$ is abelian or $G_{p}=B_{p}\left\langle a_{p}\right\rangle$, where $B_{p}$ is a normal abelian subgroup of exponent $p^{k}$, and there is a positive integer $t$ such that $t=1+p^{m}$, for some $m \leq k \leq m+d$, where $p^{d}=\left|G_{p} / B_{p}\right|$, and $a_{p}^{-1} b a_{p}=b^{t}$ for all $b \in B_{p}$; and

(2) if $p=2$, then either $G_{p}$ is a Dedekind group or $G_{p}=B_{p}\left\langle a_{p}\right\rangle$, where $B_{p}$ is a normal abelian subgroup of exponent $p^{k}$, and there is a positive integer $t$ such that $t=1+p^{m}$, for some $2 \leq m \leq k \leq m+d$, where $p^{d}=\left|G_{p} / B_{p}\right|$, and $a_{p}^{-1} b a_{p}=b^{t}$ for all $b \in B_{p}$.

Note that in both cases $G_{p}$ is nilpotent, and bounded in the non-abelian case.

Proof. By Corollary 2.4, every subgroup of $G$ is permutable in $G$ and it suffices to apply the description of the periodic locally nilpotent groups, in which every subgroup is permutable (see [17, Theorem 2.4.14]).

The next result shows a local criterion of permutability, which has some fruitful consequences.

Lemma 2.6 Suppose that a group $G$ has a local system of subgroups $\mathcal{L}$ such that if $L \in \mathcal{L}$, then every subgroup of $L$ is permutable in $L$. Then every subgroup of $G$ is permutable in $G$.

Proof. Let $H$ be an arbitrary subgroup of $G$. It suffices to prove that $\langle x\rangle H=H\langle x\rangle$ for each element $x \in G$. Let $h \in H$. Then there exists a member $L \in \mathcal{L}$ such that $x, h \in L$. In particular, $U=H \cap L \neq\langle 1\rangle$. It follows that $U$ is a permutable subgroup of $L$, and thus $\langle x\rangle U=U\langle x\rangle$. In particular, $x^{h}=u x^{k}$ for some $u \in U \leq H$ and $k \in \mathbb{Z}$. Therefore $\langle x\rangle H=H\langle x\rangle$, as required.

Corollary 2.7 Let $G$ be a group and let $\mathcal{M}$ be a family of subgroups linearly ordered by inclusion whose union is $G$ itself. Suppose that if $L \in \mathcal{M}$, then every subgroup of $L$ is permutable in $L$. Then every subgroup of $G$ is permutable in $G$.

Corollary 2.8 Let $G$ be a group having an ascending series of subgroups

$$
\langle 1\rangle=D_{0} \leq D_{1} \leq \cdots D_{\alpha} \leq D_{\alpha+1} \leq \cdots \leq D_{\gamma}=G
$$

where $\gamma$ is a limit ordinal. Suppose that every subgroup of $D_{\alpha}$ is permutable in $D_{\alpha}$ for all $\alpha<\gamma$. Then every subgroup of $G$ is permutable in $G$.

Corollary 2.9 Let $G$ be a periodic AP-group. If $G$ is a Gruenberg group, then every subgroup of $G$ is permutable. In particular, $G$ is the direct product of nilpotent Sylow subgroups. 
Proof. Let $K$ be an arbitrary finitely generated subgroup of $G$. Then $K$ is ascendant in $G$ and hence it is permutable in $G$. By the same reason every subgroup of $K$ is permutable in $G$, and hence in $K$. By Lemma 2.6 every subgroup of $G$ is permutable in $G$.

Lemma 2.10 Let $G$ be a group and let $H$ be a periodic subgroup of $G$. If $H$ is ascendant in $G$, then $H \leq O_{\pi}(G)$, where $\pi=\Pi(H)$.

Proof. It suffices to apply the formula (10) of [14, p. 20].

The following result is devoted to establish sufficient conditions for a group to be an $A P$-group.

Lemma 2.11 Let $G$ be a periodic group and let $L$ be a normal subgroup satisfying the following conditions:

(i) every subgroup of $L$ is $G$-invariant;

(ii) $G / L$ is an $A P$-group; and

(iii)] $\Pi(L) \cap \Pi(G / L)=\emptyset$.

Then $G$ is an $A P-$ group.

Proof. Let $H$ be an ascendant subgroup of $G$. Let $D=L \cap H$; by (i), $D$ is normal in $G$. Therefore it suffices to prove that $H / D$ is permutable in $G / D$. There is no loss if we assume that $D=\langle 1\rangle$, that is $H \cap L=\langle 1\rangle$. By Lemma $2.10, H \leq O_{\pi}(G)$, where $\pi=\Pi(H)$. Let $\sigma=\Pi(L)$. Then $[H, L] \leq O_{\pi}(G) \cap O_{\sigma}(G)=\langle 1\rangle$ since $\pi \cap \sigma=\emptyset$ by (iii). Then $[H, L]=\langle 1\rangle$ and it suffices to show that $\langle g\rangle H=H\langle g\rangle$, where $g$ is a $p$-element and $p$ is an arbitrary prime.

If $p \in \sigma$, then $g \in L$ and $\langle g\rangle H=\langle g\rangle \times H$. Suppose now that $p \notin \sigma$, and put $T=\langle H, g\rangle$. By Lemma 2.10, $H \leq O_{\pi}(G)$. Let $\omega=\pi \cup\{p\}$, then $\Pi(T)=\omega$ and, by (iii), $T \cap L=\langle 1\rangle$. By (ii), $G / L$ is an $A P$-group. Since the subgroup $H L / L$ is ascendant in $G / L$, it is permutable in $G / L$. In particular, $(H L / L)\langle g L\rangle=\langle g L\rangle(H L / L)$. In other words, for each element $h \in H$ and every positive integer $k$, there are an element $u \in H$ and a positive integer $m$ such that $h g^{k} L=g^{m} u L$. It follows that $u^{-1} g^{-m} h g^{k} \in L$. On the other hand $u^{-1} g^{-m} h g^{k} \in T$, that is $u^{-1} g^{-m} h g^{k} \in L \cap T=\langle 1\rangle$. Therefore $h g^{k}=g^{m} u$ and hence $H\langle g\rangle=\langle g\rangle H$, which proves the result.

The next results are mainly devoted to prove that a radical hyperfinite $A P$-group is hypercyclic.

Proposition 2.12 Let $P$ be a locally finite $p$-group, where $p$ is a prime, and let $G$ be a finite subgroup of Aut $(P)$. If $p \notin \Pi(G)$, then $P=C_{P}(G)[P, G]$. Moreover, if $P$ is abelian, then $P=C_{P}(G) \times[P, G]$. 
Proof. Let $\mathcal{L}$ be the local system of finite subgroups of $P$ and put $\mathcal{M}=$ $\left\{L^{G} \mid L \in \mathcal{L}\right\}$. Since $G$ is finite, every member $B \in \mathcal{M}$ is also finite. By $[8$, Theorem 5.3.5],

$$
B=C_{B}(G)[B, G] .
$$

If $C \in \mathcal{M}$ satisfies $B \leq C$, then $C_{B}(G) \leq C_{C}(G)$ and $[B, G] \leq[C, G]$. These inclusions give that

$$
C_{P}(G)=\bigcup_{B \in \mathcal{M}} C_{B}(G) \text { and }[P, G]=\bigcup_{B \in \mathcal{M}}[B, G] .
$$

It follows that $P=C_{P}(G)[P, G]$.

If $P$ is abelian, then $B=C_{B}(G) \times[B, G]$ (see [8, Theorem 5.2.3]). It follows that $C_{P}(G) \cap[P, G]=\langle 1\rangle$, and therefore $P=C_{P}(G) \times[P, G]$.

Lemma 2.13 Let $G$ be an $A P$-group and let $g$ be an element of $G$. Suppose that $H$ is an ascendant $\langle g\rangle$-invariant subgroup of $G$ such that $\langle g\rangle \cap H=\langle 1\rangle$. If $H$ is a Gruenberg group, then $\langle h\rangle^{\langle g\rangle}=\langle h\rangle$ for each element $h \in H$.

Proof. Put $L=\langle h\rangle^{\langle g\rangle}$. Then $\langle g, h\rangle=L \rtimes\langle g\rangle$ is a semidirect product. Since $\langle h\rangle$ is ascendant in $H$ (and hence in $G$ ), $\langle h\rangle$ is permutable in $G$. Thus $\langle g, h\rangle=\langle g\rangle\langle h\rangle$ and

$$
L=L \cap(\langle h\rangle\langle g\rangle)=\langle h\rangle(L \cap\langle g\rangle)=\langle h\rangle .
$$

Lemma 2.14 Let $G$ be a periodic AP-group and let $g$ be an element of $G$. Suppose that $H$ is an ascendant $\langle g\rangle$-invariant $p$-subgroup of $G$, where $p$ is a prime. If $H$ is a Gruenberg group and $g \notin C_{G}(H)$ but $g^{n} \in C_{G}(H)$ for some $p^{\prime}-$ number $n$, then $H$ is abelian and $[H, g]=H$.

Proof. We have $g=b x$, where $b$ is a $p$-element and $x$ is a $p^{\prime}$-element. Clearly $b \in \mathrm{C}_{G}(H)$, so $h^{g}=h^{x}$ for each element $h \in H$. Let $L$ be an arbitrary subgroup of $H$ and let $K=L^{\langle x\rangle}$. Being a Gruenberg group, $H$ is locally nilpotent. In particular, $L$ is a finite nilpotent group. Then $K$ is finite and $\langle x\rangle$-invariant. By Lemma 2.13, $x$ induces on $K$ a non-identity power automorphism. It follows from [9, Hilfssatz 5] that $K$ is abelian. Hence every finite subgroup of $H$ is abelian and, therefore, $H$ is abelian. Applying Proposition 2.12, we obtain the direct decomposition

$$
H=C_{H}(g) \times[H,\langle g\rangle]=C_{H}(g) \times[H, g] .
$$

By the choice of $g$, we have $H \neq C_{H}(g)$, and thus $[H, g] \neq\langle 1\rangle$. Suppose that $C_{H}(g) \neq\langle 1\rangle$ and choose in $C_{H}(g)$ an element $c$ of order $p$. Let $a$ be an 
element of $[H, g]$ having order $p$. Since $a \notin C_{H}(G), a^{g}=a^{d}$, where $d$ is a $p^{\prime}$-number, and moreover $d \not \equiv 1(\bmod p)$. We have

$$
(a c)^{g}=a^{g} c^{g}=a^{d} c
$$

and, since $a c \notin C_{H}(G)$, we have that

$$
(a c)^{g}=(a c)^{t},
$$

where $t$ is also a $p^{\prime}$-number such that $t \not \equiv 1(\bmod p)$. Hence

$$
a^{d} c=(a c)^{t}=a^{t} c^{t},
$$

and therefore $d \equiv t(\bmod p)$ and $t \equiv 1(\bmod p)$. This contradiction proves that $C_{H}(g)=\langle 1\rangle$ and hence $[H, g]=H$.

Lemma 2.15 Let $G$ be a periodic $A P$-group and let $H$ be a normal $p$-subgroup of $G$, where $p$ is a prime. Suppose that $H$ is a Gruenberg group. If $Q C_{G}(H) / C_{G}(H)$ is a non-identity $p^{\prime}$-subgroup, then $H$ is abelian and $[H, Q]=H$. In particular, $[H, G]=H$. Moreover, every subgroup $L$ of $H$ is $Q$-invariant and $[L, Q]=L$.

We are now in a position to show the mentioned hypercyclicity of a radical hyperfinite $A P$-group.

Lemma 2.16 Let $G$ be a radical hyperfinite group. If $G$ is an AP-group, then $G$ is hypercyclic.

Proof. Since $G$ is hyperfinite and radical, $G$ has a normal finite abelian $p$-subgroup for some prime $p$. It follows that $G$ contains a minimal normal abelian $p$-subgroup $A$. The finiteness of $A$ yields that $G / C_{G}(A)$ is finite. Being soluble, by Hall's theorem,

$$
G / C_{G}(A)=\left(Q / C_{G}(A)\right)\left(P / C_{G}(A)\right),
$$

where $Q / C_{G}(A)$ is the Hall $p^{\prime}$-subgroup of $G / C_{G}(A)$ and $P / C_{G}(A)$ is the Sylow $p$-subgroup of $G / C_{G}(A)$. Since $P / C_{G}(A)$ is a finite $p$-group, $C_{A}(P)=$ $B \neq\langle 1\rangle$. Moreover, $B$ is a $P$-invariant subgroup of $A$. By Lemma 2.13, $B$ is also $Q$-invariant. The equation $G=P Q$ yields that $B$ is $G$-invariant. However, in this case $B=A$. This shows that $G / C_{G}(A)$ is a $p^{\prime}$-group. Application of Lemma 2.13 again gives that $A$ is cyclic. Hence $G$ has a non-identity normal cyclic subgroup. From this statement and making use of transfinite induction, we obtain that $G$ is hypercyclic, as required. 


\section{Structure of hyperfinite $A P$-groups}

As the title indicates, the first objective of this section is to determine the structure of a (radical) hyperfinite $A P$-group. We split the proof of our main theorem in several results.

Lemma 3.1 Let $G$ be a radical hyperfinite group and suppose that $G$ has a normal Sylow $p$-subgroup $P$ such that $G / P$ is locally nilpotent, where $p$ is a prime. If $G$ is an $A P$-group and $G$ is not locally nilpotent, then $P$ is abelian. Moreover, if $B$ is a subgroup of $P$, then $B$ is $G$-invariant and $B=[B, G]$.

Proof. Being hyperfinite, $P$ is hypercentral. Then every subgroup of $P$ is ascendant (see, for example $[12, \S 63]$ ). By Corollary 2.4 , every subgroup of $P$ is permutable. Suppose that $P$ is non-abelian and $G=P C_{G}(P)$. Then $G=P \times Q$, where $Q$ is the Sylow $p^{\prime}$-subgroup of $G$ ([5, Theorem 7]). It follows that $G$ is locally nilpotent This is a contradiction that proves that $G \neq P C_{G}(P)$. By Lemma 2.15, $P$ must be abelian.

Reasoning as above we can see that $G \neq P C_{G}(P)$. Let $B$ be an arbitrary subgroup of $P$. Applying again Lemma 2.15, we obtain that $B$ is $G$-invariant and $[B, G]=B$.

Corollary 3.2 Let $G$ be a radical hyperfinite $A P$-group, and suppose that $G$ is not locally nilpotent. If $L$ is the locally nilpotent residual of $G$, then every Sylow p-subgroup of $G$ is abelian for each $p \in \Pi(L)$. In particular, $L$ is abelian. Moreover, every Sylow p-subgroup of $L$ is the Sylow $p$-subgroup of $G$ for each $p \in \Pi(L)$.

Proof. By Lemma 2.16, $G$ is hypercyclic. Therefore $[G, G]$ is hypercentral (see $[12, \S 64]$ ). The obvious inclusion $L \leq[G, G]$ implies that $L$ is likewise hypercentral. Then

$$
L=\operatorname{Dr}_{p \in \Pi(L)} L_{p},
$$

where $L_{p}$ is the Sylow $p$-subgroup of $L$. Pick $p \in \Pi(L)$ and put $R=$ $\operatorname{Dr}_{r \neq p} L_{r}$. Since $G$ is locally finite, $G / L$ is locally nilpotent. Thus $G / L$ has an unique Sylow $p$-subgroup, say $P / L$. It follows that $P / R$ is the unique Sylow $p$-subgroup of $G / R$. Since $R \neq L, G / R$ is not locally nilpotent. By Lemma 3.1, $P / R$ is abelian. Since $P / R$ is the unique Sylow $p$-subgroup of $G / R, L_{p} R / R \leq P / R$, and thus

$$
L_{p} \cong L_{p} /\left(L_{p} \cap R\right) \cong L_{p} R / R
$$

and $L_{p}$ is abelian. Since this holds for each $p \in \Pi(L), L$ is abelian too.

Suppose that $L_{p} R / R=L / R \neq P / R$. Being hyperfinite and locally nilpotent, $G / L$ is hypercentral. It follows that $P / L \cap \zeta(G / L)=Z / L \neq\langle 1\rangle$. 
Then $[Z / R, G / R] \leq L / R$. We have already noted that $G / R$ is not locally nilpotent. Since $Z / R$ is a $G$-invariant subgroup of $P / R$, Lemma 3.1 yields that $[Z / R, G / R]=Z / R$. This contradiction proves the equation $L_{p} R / R=$ $L / R=P / R$. It follows that $L_{p}$ is the Sylow $p$-subgroup of $G$.

Lemma 3.3 A radical hyperfinite $A P$-group $G$ is metabelian.

Proof. Let $L$ be the locally nilpotent residual of $G$. Since $L \leq[G, G]$,

$$
G /[G, G] \cong(G / L) /([G, G] / L) .
$$

Since $G / L$ is locally nilpotent,

$$
G / L=\operatorname{Dr}_{p \in \Pi(G / L)} S_{p} / L,
$$

where $S_{p} / L$ is the Sylow $p$-subgroup of $G / L$. Then

$$
[G, G] / L=[G / L, G / L]=\operatorname{Dr}_{p \in \Pi(G / L)}\left[S_{p} / L, S_{p} / L\right] .
$$

Put $D_{p} / L=\left[S_{p} / L, S_{p} / L\right]$. Being hyperfinite, $G / L$ is hypercentral. In particular, every subgroup of $G / L$ is ascendant in $G / L$ (see $[12, \S 63]$ ). Corollary 2.5 yields that every Sylow $p$-subgroup $S_{p} / L$ of $G / L$ is metabelian, so that $D_{p} / L$ is abelian for each $p \in \Pi(G / L)$.

On the other hand, by Lemma $2.16, G$ is hypercyclic. Therefore $[G, G]$ is hypercentral (see[12, §63]). Pick $p \in \Pi([G, G]) \backslash \Pi(L)$ and choose the Sylow $p$-subgroup $P$ of $[G, G]$. By Corollary 3.2, $P \cap L=\langle 1\rangle$. Therefore

$$
P \cong P(P \cap L) \cong P L / L \leq D_{p} / L,
$$

and then $P$ is abelian. By Corollary 3.2, the Sylow $p$-subgroup of $L$ is abelian for each $p \in \Pi(L)$. It follows that $[G, G]$ is abelian.

Lemma 3.4 Let $G$ be a radical hyperfinite group and let $R$ be the locally nilpotent radical of $G$. If $G$ is an $A P$-group, then $R=L \times Z$, where $L$ is the locally nilpotent residual of $G$ and $Z$ is the upper hypercenter of $G$.

Proof. By Corollary 3.2, $L$ is an abelian normal subgroup of $G$, so that $L \leq R$. Being locally nilpotent, $R$ is the product of its Sylow subgroups. Let $\pi=\Pi(L)$. By Corollary 3.2, $L$ is the Hall $\pi$-subgroup of $R$. Hence $R=L \times Z$, where $Z$ is the Hall $\pi^{\prime}$-subgroup of $R$. Since the factor group $G / L$ is locally nilpotent and hyperfinite, it is hypercentral. Let

$$
\langle 1\rangle=C_{0} / L \leq C_{1} / L \leq \cdots \leq C_{\alpha} / L \leq C_{\alpha+1} / L \leq \cdots C_{\gamma} / L=G / L
$$

be the upper central series of $G / L$. Let $Z_{\alpha}$ be the subgroup of $Z$ such that $Z_{\alpha} L / L=C_{\alpha} / L \cap Z L / L$, for every $\alpha<\gamma$. We have

$$
\left[Z_{\alpha+1}, G\right] L=\left[Z_{\alpha+1} L / L, G / L\right] \leq C_{\alpha} / L \cap Z L / L=Z_{\alpha} L / L,
$$


so that

$$
\left[Z_{\alpha+1}, G\right] \leq Z_{\alpha} L \cap Z=Z_{\alpha}(L \cap Z)=Z_{\alpha}
$$

for all $\alpha<\gamma$. In other words, the series

$$
\langle 1\rangle=Z_{0} \leq Z_{1} \leq \cdots \leq Z_{\alpha} \leq Z_{\alpha+1} \leq \cdots Z_{\gamma}=Z
$$

is $G$-central. This shows that the upper hypercenter $\zeta_{\infty}(G)$ of $G$ contains $Z$. On the other hand, $\zeta_{\infty}(G) \leq R$, and then

$$
\zeta_{\infty}(G)=Z \times\left(\zeta_{\infty}(G) \cap L\right)
$$

We claim that $\zeta_{\infty}(G) \cap L=\langle 1\rangle$. For, otherwise $\zeta(G) \cap L=Y \neq\langle 1\rangle$. Pick $p \in \Pi(Y)$, and let $P$ be the Sylow $p$-subgroup of $L$ and let $Q$ be the Hall $p^{\prime}$ subgroup of $L$. By Corollary 3.2, $P$ is abelian. If we suppose that $C_{G}(P)=$ $G$, then $C_{G / Q}(P Q / Q)=G / Q$. It follows that $G / Q=P Q / Q \times S / Q$, where $S / Q$ is the Hall $p^{\prime}$-subgroup of $G / Q$ ([5, Theorem 7]). This means that the factor-group $G / Q$ is hypercentral, which contradicts the choice of $L$. This contradiction shows that $C_{G}(P) \neq G$, so there is a $p^{\prime}$-element $g$ such that $g \notin C_{G}(P)$. By Proposition 2.12, $P=C_{P}(g) \times[P, g]$. By the choice of $P$, we have that $C_{P}(g) \neq\langle 1\rangle$. Therefore $[P, g] \neq P$. However Lemma 2.15 yields that $[P, g]=P$. This contradiction shows our claim, that is $\zeta_{\infty}(G) \cap L=\langle 1\rangle$. Therefore $\zeta_{\infty}(G)=Z$, as required.

We are now in a position to prove our result on the description of the structure of radical hyperfinite $A P$-groups.

Theorem 3.5 Let $G$ be a radical hyperfinite AP-group. Then the following assertions hold:

(i) $G$ is metabelian;

(ii) if $R$ is the locally nilpotent radical of $G$, then $R=L \times Z$, where $L$ is the locally nilpotent residual of $G$ and $Z$ is the upper hypercenter of $G$;

(iii) The Sylow p-subgroup of $L$ is the Sylow p-subgroup of $G$ for every $p \in \Pi(L)$;

(iv) every subgroup of $L$ is $G$-invariant; and

(v) $G / L$ is hypercentral and every subgroup of $G / L$ is permutable.

Moreover, if the factor group $G / L$ is countable, then $G$ splits over $L$. In particular, if $G$ is countable, then $G$ splits over $L$.

On the other hand, if $G$ is a periodic group having a normal abelian subgroup $L$ that satisfies the conditions (iii)-(v), then $G$ is an AP-group.

Proof. The statement (i) follows from Lemma 3.3, the statement (ii) follows from Lemma 3.4 and the statement (iii) follows from Corollary 3.2. As we see above, $C_{G}(L) \neq G$, so that $G / C_{G}(L)$ is a non-identity group such that $\Pi(L) \cap \Pi(G / L)=\emptyset$. Therefore, Lemma 2.15 yields (iv). 
Finally $G / L$ is locally nilpotent and, being hyperfinite, $G / L$ is hypercentral. In particular, every subgroup of $G / L$ is ascendant in $G / L$ (see $[12, \S 63])$. Hence every subgroup of $G / L$ is permutable, that is (v) holds. To obtain the mentioned splitting of $G$ over $L$ it suffices to apply [6, Theorem 2.4.5], for example.

The last assertion is an immediate consequence of Lemma 2.11.

We remark that the description of hypercentral periodic groups, every subgroup of which is permutable has been obtained above in Corollary 2.5. Since a radical hyperfinite $A P$-group $G$ is hypercyclic by Lemma 2.16 , it is not hard to see that $G$ has a descending Sylow series, that is $G$ has a series

$$
G=G_{0} \geq G_{1} \geq \cdots \geq G_{n} \geq G_{n+1} \geq \cdots \bigcap_{n \in \mathbb{N}} G_{n}=\langle 1\rangle
$$

of normal subgroups satisfying the following conditions:

(i) for every $n \in \mathbb{N}, G_{n} / G_{n+1}$ is a $p_{n}$-group, for some prime $p_{n}$; and

(ii) $p_{0}<p_{1}<\cdots<p_{n}<p_{n+1}<\cdots$.

As a consequence, we can see that $2 \notin \Pi(L)$. Another consequence is the following important result.

Corollary 3.6 Let $G$ be a radical hyperfinite AP-group. Then every subgroup of $G$ is an $A P-$ group.

The corresponding result for $P T$-groups is not true as the locally dihedral 2 -group shows. In this sense, we say that a group $G$ is a $\overline{P T}$-group if every subgroup of $G$ is a $P T$-group, that is, if for subgroups $H, K$ and $L$ such that $H$ is permutable in $K$ and $K$ is permutable in $L$, it is always true that $H$ is permutable in $L$. It is easy to prove, using transfinite induction, that every ascendant subgroup of a $\overline{P T}$-group is permutable. Since every $A P$-group is a $P T$-group, we also deduce from Corollary 3.6 that $\overline{P T}$-groups are exactly $A P$-groups in the universe of all radical hyperfinite groups.

We recall that a group $G$ is said to be a $T$-group if normality is a transitive relation in $G$, that is if $K$ is a normal subgroup of $H$ and $H$ is a normal subgroup of $G$ implies that $K$ is normal in $G$. The study of infinite $T$-groups has began in a paper of D. J. S. Robinson [13]. This study has been continued in many papers, where infinite $T$-groups have been studied from different points of view. The next results show connections between the classes of $A P$-groups and $T$-groups.

Corollary 3.7 Let $G$ be a radical hyperfinite AP-group. If the Sylow 2-subgroups of $G$ are Dedekind groups and the Sylow p-subgroups of $G$ are abelian for $p \neq 2$, then $G$ is a metabelian $T$-group. 
Proof. Let $L$ be the locally nilpotent residual of $G$. If $H$ is a given subnormal subgroup of $G$, we put $D=L \cap H$. By the condition (i) of Theorem 3.5, $D$ is normal in $G$. Therefore it suffices to prove that $H / D$ is normal in $G / D$. Without loss of generality, we can suppose that $D=\langle 1\rangle$, that is $H \cap L=\langle 1\rangle$. By Lemma 2.10, $H \leq O_{\pi}(G)$, where $\pi=\Pi(H)$. Put $\sigma=\Pi(L)$. It follows that $[H, L] \leq O_{\pi}(G) \cap O_{\sigma}(G)=\langle 1\rangle$ since $\pi \cap \sigma=\emptyset$ by the condition (iii) of Theorem 3.5. Then $[H, L]=\langle 1\rangle$. It suffices to show that $g^{-1} H g=H$, where $g$ is a $p$-element and $p$ is an arbitrary prime. If $p \in \sigma$, then $g \in L$ and $\langle g\rangle H=H\langle g\rangle$. In particular, $g^{-1} H g=H$. Suppose now that $p \notin \sigma$ and put $R=\langle H, g\rangle$. By Lemma 2.10, $H \leq O_{\pi}(G)$. Let $\omega=\pi \cup\{p\}$ so that $\Pi(R)=\omega$ and, by the condition (iii) of Theorem 3.5, $R \cap L=\langle 1\rangle$. Since $G / L$ is Dedekind, $H L / L$ is normal in $G / L$. Hence for each element $h \in H$ we have $[h L, g L]=[h, g] L \in H L / L$, that is $[h, g] \in H L$. On the other hand, $g, h \in R$ and so $[h, g] \in R$. Thus $[h, g] \in H L \cap R=H(L \cap R)=H$. This means that $g^{-1} H g=H$, which proves the result.

Corollary 3.8 Let $G$ be a radical hyperfinite AP-group. If the Sylow p-subgroups of $G$ are abelian for all primes $p \in \Pi(G)$, then $G$ is a metabelian T-group.

Corollary 3.9 Let $G$ be a radical hyperfinite AP-group. If the locally nilpotent residual of $G$ coincides with the derived subgroup, then $G$ is a metabelian T-group.

Proof. Let $p$ be a prime, and let $P$ be a Sylow $p$-subgroup of $G$. Let $L$ be the locally nilpotent residual of $G$. If $p \in \Pi(L)$, then by the condition (iii) of Theorem 2.7, $P \leq L$, and the condition (iv) of Theorem 3.5, yields that $P$ is abelian. If $p \notin \Pi(L)$, then, by the condition (iii) of Theorem 3.5, $P \leq L=\langle 1\rangle$. Since $G / L$ is abelian, $P$ is abelian too. It suffices to apply Corollary 3.8.

Corollary 3.10 Let $G$ be a radical hyperfinite AP-group. If $\zeta(G) \cap[G, G]$ $=\langle 1\rangle$, then $G$ is a metabelian $T$-group.

Proof. Let $R$ be the locally nilpotent radical of $G$. By the condition (ii) of Theorem 3.5, $R=L \times Z$, where $L$ is the locally nilpotent residual of $G$, and $Z$ is the upper hypercenter of $G$. Lemma 2.16 yields that $G$ is hypercyclic. It follows that $[G, G]$ is hypercentral (see $[12, \S 64]$ ). In particular, $[G, G] \leq R$. On the other hand, $L \leq[G, G]$, so that

$$
[G, G]=L \times([G, G] \cap Z)=L
$$

and it suffices to apply Corollary 3.9.

Corollary 3.11 Let $G$ be a radical hyperfinite AP-group. If $\zeta(G)=\langle 1\rangle$, then $G$ is a metabelian T-group. 


\section{The structure of $A P$-groups in some wider classes of groups}

In this section we extend some of our previous results to certain extensions of the class of radical hyperfinite groups. They are the hyperabelian groups, hyper- $N$-groups, hyper-Gruenberg groups. In general, these classes of groups are much wider than the class of radical hyperfinite groups. Really the class of hyperfinite groups is "very narrow". In particular, there are hyperabelian (even soluble) groups, which are not hyperfinite. In fact, for every prime $p$ and every periodic locally cyclic $p^{\prime}$-group $L$ there exists a simple $\mathbb{F}_{p} L$-module $A$, where $\mathbb{F}_{p}$ is the field of order $p$ (see, for example, [11, Chapter 2]). The natural semidirect product of $A$ by $L$ gives an example of a metabelian group which is not hyperfinite. However, as we will see in this section, periodic $A P$-groups in the above classes are hyperfinite.

Lemma 4.1 Let $G$ be an AP-group and let $P$ be a normal p-subgroup of $G$ for some prime $p$. Suppose that $P$ has an ascending series of normal subgroups

$$
\langle 1\rangle=D_{0} \leq D_{1} \leq \cdots \leq D_{\alpha} \leq D_{\alpha+1} \leq \cdots D_{\gamma}=P
$$

whose factors are $N$-groups. Then every subgroup of $P$ is permutable in $G$.

Proof. We proceed by induction on $\gamma$. If $\gamma=1$, then $P$ itself is a $N$-group. In this case every subgroup of $P$ is ascendant in $P$ and hence in $G$. It follows that every subgroup of $P$ is permutable in $G$.

Let now $\gamma>1$ and suppose that we have already proved that every subgroup of $D_{\alpha}$ is permutable in $G$ for all $\alpha<\gamma$. If $\gamma$ is a limit ordinal, then the result follows from Corollary 2.8. Therefore we assume that $\gamma-1$ exists. Put $D=D_{\gamma-1}$. By induction, every subgroup of $D$ is permutable in $G$. Being a $p$-group, $D$ is hypercentral (see [17, Theorem 2.4.14]). Since every subgroup of $P / D$ is ascendant in $P / D$, Corollary 2.4 yields that every subgroup of $P / D$ is permutable in $G / D$ and so $P / D$ is likewise hypercentral. Let $\mathcal{L}$ be the family of all finite subgroups of $P / D$ and let $U$ and $V$ be $P$-invariant subgroups of $D$ such that $U \leq V$ and the factor $V / U$ is $D$ central. If $L / D \in \mathcal{L}$, then $L=K D$ for some finite subgroup $K$ of $P$. Pick $v \in V$, and put $T=\langle v, K\rangle$. Being a finite $p$-subgroup, $T$ is nilpotent and then $T U / U$ is nilpotent too. Since $V$ is normal in $P$,

$$
Z / U=V / U \cap \zeta(T / U) \neq\langle 1\rangle
$$

This shows that

$$
C_{V / U}(L / D) \neq\langle 1\rangle
$$


Applying transfinite induction, we obtain that $V / U$ has an ascending $L$-central series. Since this holds for each factor of the upper central series of $D, L$ is hypercentral. Since $L$ is ascendant in $G$, every subgroup of $L$ is ascendant in $G$. Applying Lemma 2.6, we see that every subgroup of $P$ is permutable in $G$, as required.

Lemma 4.2 Let $G$ be a locally finite group. Suppose that, for some prime $p$, $G$ has a normal Sylow $p$-subgroup $P$ such that $P$ does not contain $C_{G}(P)$. Then $O_{p^{\prime}}(G) \neq\langle 1\rangle$. Moreover, if $G / P$ is infinite and $G / C_{G}(P)$ is finite, then $O_{p^{\prime}}(G)$ is infinite.

Proof. Put $C=C_{G}(P)$ and $A=P \cap C$. The isomorphism

$$
C / A=C /(C \cap P) \cong C P / P
$$

shows that $C / A$ is a $p^{\prime}$-group. It follows that $A$ is the Sylow $p$-subgroup of $C$ and $C=A \times O_{p^{\prime}}(C)$ ([5, Theorem 7]). The conditions of the Lemma imply that $C \neq A$, therefore $O_{p^{\prime}}(C) \neq\langle 1\rangle$. Since $C$ is normal in $G, O_{p^{\prime}}(C) \leq$ $O_{p^{\prime}}(G)$, and the first assertion follows. The second one is immediate.

Lemma 4.3 Let $G$ be a periodic AP-group. Suppose that $O_{p^{\prime}}(G)=\langle 1\rangle$ for some prime $p$. If $G$ is a hyper-N-group, then $G / O_{p}(G)$ is finite.

Proof. Obviously we can suppose that $G$ is infinite. Put $P=O_{p}(G)$. Suppose the contrary, that is $G / P$ is infinite. We claim that $P$ is infinite. For, otherwise, $O_{p^{\prime}}(G)$ is infinite by Lemma 4.2, which is impossible. Hence $P$ is infinite. Since $G$ is a hyper $-N$-group, then we fall into one of the following cases.

(a) $G$ has a finite series of normal subgroups

$$
P=D_{0} \leq D_{1} \leq \cdots \leq D_{n} \leq D_{n+1},
$$

where the factors $D_{j} / D_{j-1}$ are finite $r_{j}$-groups for some prime $r_{j}, 1 \leq j \leq n$, and $D_{n+1} / D_{n}$ is an infinite $q$-group for some prime $q$.

(b) $G$ has an infinite series of normal subgroups

$$
P=D_{0} \leq D_{1} \leq \cdots \leq D_{n} \leq D_{n+1} \leq \cdots,
$$

where the factors $D_{j} / D_{j-1}$ are finite $r_{j}$-groups for some prime $r_{j}, 1 \leq j$.

Consider first the case (a). Applying Lemma 4.2, after finitely many steps, we see that $G$ has a normal subgroup $Q$ such that $P \leq Q$ and $Q / P$ is an infinite $q$-group. By definition of $P$, we have $p \neq q$. By Lemma 4.1, every subgroup of $P$ is permutable in $G$. Then Corollary 2.5 yields that either $P$ is abelian or $P=B\langle b\rangle$, for some element $b$ and some abelian normal subgroup $B$ of $P$ such that every cyclic subgroup of $B$ is $P$-invariant. In both cases, Lemma 2.13 proves that every subgroup of $A$ (respectively of $B$ ) is $Q$-invariant, while, in particular, in the second case $B$ is normal in $Q$. 
If $P$ is abelian, then $Q / C_{Q}(P)$ is finite (see [17, Theorem 1.5.6]). Applying Lemma 4.2 in this case, $O_{p^{\prime}}(G)=\langle 1\rangle$, which is impossible. Now we suppose that $P$ is non-abelian. Since $P / B$ is finite, by Lemma 4.2, $R / B=O_{p^{\prime}}(Q / B)$ is infinite. Repeating now the same arguments that we used when supposed $P$ abelian, we obtain again that $O_{p^{\prime}}(G) \neq\langle 1\rangle$. Therefore the case (a) leads to a contradiction.

Consider now the case (b). Put $D=\bigcup_{n \in \mathbb{N}} D_{n}$. Since $D$ is normal in $G, D$ is an $A P$-group by Lemma 2.2. Being hyperfinite, $D / P$ is metabelian by Theorem 3.5. Let $L / P$ be the locally nilpotent radical of $D / P$. If we assume that $L / P$ is finite, then $D / P$ is finite, because $C_{D / P}(L / P) \leq L / P$. However this contradicts our assumption. Hence $L / P$ is infinite. By definition of $P, L / P$ is a $p$-group. Reasoning as in case (a), we obtain again that $O_{p^{\prime}}(G) \neq\langle 1\rangle$. As in case (a), this is a contradiction. This contradiction shows that $G / P$ is finite, as required.

Theorem 4.4 Let $G$ be a periodic AP-group. If $G$ is a hyper-N-group, then $G$ is hyperfinite.

Proof. Let

$$
\langle 1\rangle=D_{0} \unlhd D_{1} \unlhd \cdots \unlhd D_{\alpha} \unlhd D_{\alpha+1} \unlhd \cdots D_{\gamma}=G
$$

be an ascending series of normal subgroups of $G$ whose factors are $N$-groups. Pick $p \in \Pi\left(D_{1}\right)$, and let $S$ be the Sylow $p$-subgroup of $D_{1}$. Put $Q=O_{p^{\prime}}(G)$ and $P / Q=O_{p}(G / Q)$. By Lemma $4.3, G / P$ is finite. Then Lemma 4.1 shows that every subgroup of $P / Q$ is permutable in $G / Q$. Being a $p$-group, $P / Q$ is hypercentral by Corollary 2.5. Since $S Q / Q$ is normal in $P / Q$,

$$
S Q / Q \cap \zeta(P / Q) \neq\langle 1\rangle \text {. }
$$

Pick $1 \neq z \in S$ such that $z Q \in S Q / Q \cap \zeta(P / Q)$. Then $C_{G / Q}(z Q)$ has finite index. Put $Z=\langle z\rangle^{G}$ so that

$$
Z Q / Q \leq S Q / Q \cap \zeta(P / Q),
$$

and hence $Z Q / Q$ is finite. Since $S$ is normal in $G, Z \leq S$, which implies the finiteness of $Z$. Consequently, $G$ has a non-identity finite normal subgroup. Applying transfinite induction, we obtain that $G$ is hyperfinite.

It is worth noting that the groups in Theorem 4.4 are in fact hypercyclic by Lemma 2.16. Thus, we obtain the following consequences.

Corollary 4.5 Let $G$ be a periodic AP-group. If $G$ is a hyper-N-group, then $G$ is a hypercyclic metabelian AP-group.

Corollary 4.6 Let $G$ be a periodic AP-group. If $G$ is a hyper-Gruenberg group, then $G$ is a hypercyclic metabelian AP-group. In particular, if $G$ is a countable radical group, then $G$ is a hypercyclic metabelian group. 
Proof. Let $U \leq V$ be two normal subgroups of $G$ such that $U / V$ is a Gruenberg group. By Corollary 2.9, every Sylow $p$-subgroup of $U / V$ is nilpotent, for all primes $p$. It follows that $G$ is hypernilpotent. In particular, it is a hyper- $N$-group and we can apply Corollary 4.5 .

Corollary 4.7 Let $G$ be a periodic AP-group. If $G$ is hyperabelian, then $G$ is a hypercyclic metabelian AP-group.

Corollary 4.8 Let $G$ be a periodic AP-group. If $G$ is soluble, then $G$ is a hypercyclic metabelian AP-group.

Corollary 4.9 Let $G$ be a periodic AP-group. If $G$ is residually soluble, then $G$ is a hypercyclic metabelian AP-group.

Proof. By Lemma 2.1, every soluble factor-group of $G$ is an $A P$-group, and, by Corollary 4.8, this factor-group is metabelian. If follows that $G$ itself is metabelian. By Corollary 4.8, $G$ is also hypercyclic.

We recall that a group $G$ is called a Baer group, if every finitely generated subgroup of $G$ is subnormal in $G$. Since every Baer group is a Gruenberg group, we have

Corollary 4.10 Let $G$ be a periodic AP-group. If $G$ is a hyper-Baer group, then $G$ is a hypercyclic metabelian AP-group.

\section{The construction of periodic soluble $A P$-groups}

The main object of this section is to show how our results allows us to construct all periodic soluble $A P$-groups.

Let $G$ be a periodic soluble $A P$-group and let $R$ be the locally nilpotent radical of $G$. In particular, it follows from Theorem 4.4 that $G$ is hyperfinite. By virtue of Lemma 3.4, we have $R=L \times Z$, where $L$ is the locally nilpotent residual of $G$ and $Z$ is the upper hypercenter of $G$. It is clear then that $G$ is embedded into $G / L \times G / Z$. In addition, applying Theorem $3.5, G / L$ is a locally nilpotent group with all subgroups permutable. Consider $H=$ $G / Z$ and let $K=L Z / Z$. Then $K=\operatorname{Dr}_{p \in \Pi(K)} K_{p}$ where $K_{p}$ is the Sylow $p$-subgroup of $K$. Let $U_{p}$ be the normal subgroup of $G$ which is maximal with the property: $\operatorname{Dr}_{q \neq p} K_{q}$ is contained in $U_{p}$ and $U_{p} \cap K_{p}=1$. Now we consider the quotient $H / U_{p}$. Then $C_{H / U_{p}}\left(K_{p} U_{p} / U_{p}\right)=K_{p} U_{p} / U_{p}$. Furthermore, by Theorem 3.5, every Sylow subgroup of $L$ is the Sylow subgroup of $G$. It follows that $\left(H / U_{p}\right) /\left(K_{p} U_{p} / U_{p}\right)$ is a $p^{\prime}$-group. Since $H / U_{p}$ induces power automorphisms on $K_{p} U_{p} / U_{p}$, then being periodic, it is finite of order at most $p-1$. Hence $H / U_{p}$ is the semidirect product of $K_{p} U_{p} / U_{p}$ and $V_{p} / U_{p}$ where $V_{p} / U_{p}$ is finite of order at most $p-1$. Since the intersection of all $U_{p}$ is trivial (it lies in the centralizer of $K$ ), then $H$ is embedded in the Cartesian product of all the $\left(H / U_{p}\right)^{\prime} s$. 
Let $\pi$ be a non-empty set of primes such that $2 \notin \pi$. Let $A_{p}$ be an abelian $p$-group for every prime $p \in \pi$. We consider the natural semidirect product $C_{p}$ of $A_{p}$ and $F_{p}$ where $F_{p}$ is a finite group of order at most $p-1$ such that $F_{p}$ acts as power automorphisms group on $A_{p}$. Let $B$ be the Cartesian product of $C_{p}, p \in \pi$, and let $A$ be the direct product of $A_{p}, p \in \pi$. Choose an arbitrary periodic subgroup $P$ of $B$ including $A$ and consider $G$ the direct product of $P$ and $Q$ where $Q$ is a locally nilpotent $\pi^{\prime}$-group such that every subgroup of $Q$ is permutable. Then, by the above paragraph, every periodic soluble $A P$-group arises as a subgroup of such group.

\section{References}

[1] Alejandre, M. J., Ballester-Bolinches, A. And Pedraza-AguiLERA, M. C.: Finite soluble groups with permutable subnormal subgroups. J. Algebra 240 (2001), 705-722.

[2] Ballester-Bolinches, A., Esteban-Romero, R. and PedrazaAguilerA, M. C.: On finite groups in which subnormal subgroups satisfy certain permutability conditions. In Advances in Algebra, 38-45. World Sci. Publ., River Edge, NJ, 2003.

[3] Ballester-Bolinches, A., Kurdachenko, L. A., and Pedraza, T.: On periodic radical groups in which permutability is a transitive relation. J. Pure Appl. Algebra 210 (2007), 665-671.

[4] Beidleman, J. C., Brewster, B. and Robinson, D. J. S.: Criteria for permutability to be transitive in finite groups. J. Algebra 222 (1999), no. 2, 400-412.

[5] Chernikov, S. N.: On complementability of the Sylow subgroups in some classes of infinite groups. Math. Sbornik 37 (1955), 557-566.

[6] Dixon, M. R.: Sylow theory, formations and Fitting classes in locally finite groups. World Scientific Publishing, River Edge, NJ, 1994.

[7] Gaschütz, W.: Gruppen, in denen das Normalteilersein transitiv ist. J. Reine Angew. Math. 98 (1957), 87-92.

[8] Gorenstein, D.: Finite groups. Harper \& Row, New York-London, 1968.

[9] Huppert, B.: Zur Sylowstruktur auflösbarer Gruppen. Arch. Math. 12 (1961), 161-169.

[10] Kargapolov, M. I.: On generalized solvable groups. Algebra i Logika Sem. 2 (1963), 19-28.

[11] Kurdachenko, L. A., Otal, J. and Subbotin, I. Ya.: Groups with prescribed quotient groups and associated module theory. Series in Algebra 8. World Scientific Publishing, River Edge, NJ, 2002.

[12] Kurosh, A. G.: The theory of groups. Nauka, Moskow, 1967.

[13] Robinson, D. J.S.: Groups in which normality is a transitive relation. Proc. Cambridge Philos. Soc. 60 (1964), 21-38. 
[14] Robinson, D. J. S.: Finiteness conditions and generalized soluble groups. Part 1. Ergebnisse der Mathematik und ihrer Grenzgebiete 62. SpringerVerlag, New York-Berlin, 1972.

[15] Robinson, D. J. S.: Minimality and Sylow-permutability in locally finite groups. Ukrain. Math. J. 54 (2002), 1038-1049.

[16] Schmidt, O. Yu.: On infinite special groups. Math. Sbornik 8 (1940), 363-375.

[17] Schmidt, R.: Subgroup lattices of groups. De Gruyter Expositions in Mathematics 14. Walter de Gruyter \& Co., Berlin, 1994.

[18] Stonehewer, S. E.: Permutable subgroups of infinite groups. Math. Z. 125 (1972), 1-16

[19] ZaCher, G.: I gruppi risolubili finiti in cui i sottogruppi di composizione coincidono con i sottogruppi quasi-normali. Atti Accad. Naz. Lincei Rend. Cl. Sci. Fis. Mat. Natur. (8) 37 (1964), 150-154.

Recibido: 15 de enero de 2007 Revisado: 17 de octubre de 2007

A. Ballester-Bolinches Departament d'Àlgebra Universitat de València 46100 Burjassot (València), Spain Adolfo.Ballester@uv.es

L. A. Kurdachenko Department of Algebra National Dnepropetrovsk University 49050 Dnepropetrovsk, Ukraine

lkurdachenko@i.ua J. Otal Departamento de Matemáticas

Universidad de Zaragoza 50009 Zaragoza, Spain otal@unizar.es

T. Pedraza Departamento de Matemática Aplicada Escuela Técnica Superior de Informática Aplicada Universidad Politécnica de Valencia 46022 Valencia, Spain tapedraz@mat.upv.es

This research was supported by Proyecto MTM2004-08219-C02-02 (Ballester-Bolinches, Pedraza) and Proyecto MTM2004-04842 (Kurdachenko, Otal) of MEC (Spain) and Proyecto GV/2007/243 (Pedraza) of Generalitat (Valencia, Spain). 